\title{
Study suggests stem cell therapy for brain may be more complicated than originally thought
}

In recent years, research has focused on the use of stem cells as a therapy for many diseases. In developing embryos, the cells of the body originate from stem cells, some of which persist into adulthood. Stem cell therapies hope to utilize these cells to repair organs and tissues damaged by disease. For example, neural stem cells could be used to replace the neurons killed in neurodegenerative diseases such as Alzheimer's.

However, as Carlos E Lois (Massachusetts Institute of Technology, MA, USA) explains, stem cell therapy may prove to be more complicated than was originally thought. "In the stem cell field, it is generally thought that the main limitation to achieve brain repair is simply for the new neurons to reach a given brain region and to ensure their survival," says Lois. "Once there, it has been assumed that stem cells will 'know what to do' and will become the type of neuron that is missing. Our experiments indicate that things are much more complicated."

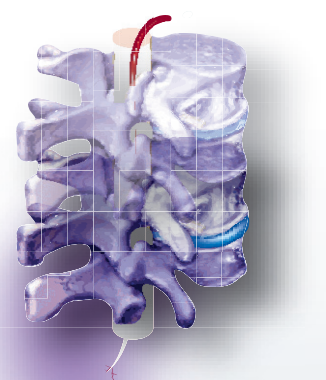

Lois and colleagues found that adult neural stem cells are preprogrammed to make defined connections and not to make others. This makes it impossible to transplant the neural stem cells to other areas, the spinal cord, for example, because they do not change the type of connections they make. "A stem cell that produces neurons that could be useful to replace neurons in the cerebral cortex (the type of neurons lost in Alzheimer's disease) will be most likely useless to replace neurons lost in the spinal cord," says Lois. Furthermore, he is of the opinion that, "...it is wishful thinking to hope that adult stem cells will be able to modify themselves so that they can become other types of neurons lost to injury or disease".

For embryonal stem cells to realize their potential to differentiate into different cell types, they have to be 'instructed' to become liver cells, heart cells, neurons and so on. Lois and colleagues studied neural stem cells and the results suggest that researchers will need to discover how to program stem cells to produce specific functioning neurons. Without such instructions, neurons will only make connection with the defined partners for which they were preprogrammed. "Moreover, because there are many different types of neurons in the cerebral cortex, it is likely that we will have to figure out how to program stem cells to become many different types of neurons, each of them with a different set of prespecified connections," explains Lois. Consequently, it seems likely "...that we will have to know much more about the different types of neuronal stem cells, and to identify the characteristic features of their progeny," he says. This is further complicated by the "...need to have access to many different types of 'tailored' stem cells that give rise to many different types of neurons with specific connections. In addition, we may need a combination of several of these tailored stem cells to eventually be able to replace the different types of neurons lost in a given brain region".

Source: Kelsch W, Mosley CP, Lin CW, Lois C: Distinct mammalian precursors are committed to generate neurons with defined dendritic projection patterns. PLoS Biol. 5(11), E300 (2007).



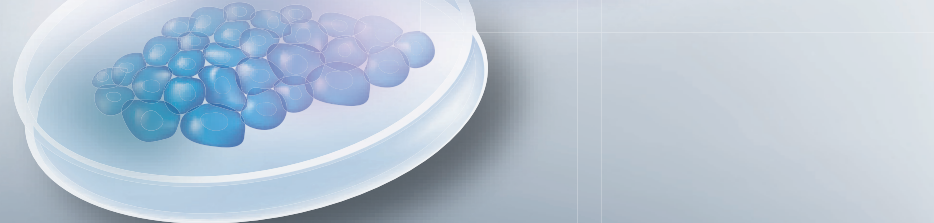




\section{Priority Paper Alerts}

Thickening in the somatosensory cortex of patients with migraine.

DaSilva AF, Granziera C, Snyder J, Hadjikhani N Neurology 69(21), 1990-1995 (2007).

The study aimed to determine the morphologic changes in the somatosensory cortex (SSC) of patients with migraine. The cortical thickness of the SSC was measured in vivo in a total of 36 subjects. This included 12 migraine with aura patients, 12 migraine without aura patients and 12 age- and sex-matched healthy subjects. The data were present as average maps of significant changes in cortical thickness. The SSCs in patients with migraine were thicker than those of healthy subjects. Differences in the thickness of the caudal SSC, where the trigeminal area is somatotopically represented, were the most significant. The SSC is important in noxious and non-noxious somatosensory processing. The study concludes that neuroplastic changes in the cortical and subcortical structure of the trigeminal somatosensory system may lead to, or be caused by repetitive migraine attacks.

\section{Associations between MDR1 gene} polymorphisms and schizophrenia and therapeutic response to olanzapine in female schizophrenic patients.

Bozina N, Kuzman MR, Medved V, Jovanovic N, Sertic J, Hotujac L: J. Psychiatr. Res. 42(2), 89-97 (2007).

The MDR1 gene encodes an efflux transporter. Variants of this gene have been implicated as disease susceptibility factors and treatment response determinants. This study aimed to determine the relationship between exon 21 G2677T and exon 26 C3435T genetic variants of MDR1 with susceptibility and treatment response in females with schizophrenia. Allele, genotype and haplotype distributions were compared in 117 female schizophrenia patients and 123 female controls. This was followed by a study of response to olanzapine treatment in 87 out of 117 previously unmedicated schizophrenia patients. The representation of the G2677/C3435 haplotype was lower in schizophrenia patients than controls. The loci were in significant linkage disequilibrium. The MDR1 exon 21 G2677T genotypes were significantly associated with treatment response; the $T$ allele and TT genotype were associated with a better treatment response. The data suggest that mutations in the MDR1 gene are associated with schizophrenia susceptibility and response to treatment in females.

A University of Manchester study suggests that faster evaluation of patients suffering from 'mini strokes' is the key to preventing stroke in high-risk patients

\section{Faster assessment of patients suffering TIAs may prevent hundreds of strokes}

Transient ischemic attacks (TIAs) or mini strokes are characterized by a temporary weakening of one side of the face and the corresponding arm. TIAs have also been shown to dramatically increase the chance of suffering a major stroke within days of the onset of symptoms. In fact, some studies have calculated the risk to be as high as a one-in-four probability.

Early assessment of patients suffering TIAs is of utmost importance, and "...current UK guidelines recommend that all people who have had a TIA should be assessed by a specialist within 7 days of the start of symptoms," says Craig Smith (University of Manchester, UK). However, a recent study by Smith and colleagues found that approximately two-thirds of patients who attended 'rapid access' TIA clinics were not seen within the recommended 7 days. In fact, in some cases access to the specialist clinics took twice as long as the guidelines recommend. "Our findings suggest that this standard is not being met and, in reality, TIA patients should ideally be assessed for risk of further stroke within a couple of days, if not on the same day as the initial symptoms," says Smith.

The study monitored 711 people who had suffered a TIA or minor stroke 15 days earlier and who attended five centers in Manchester and Liverpool. The risk of recurrent TIA, stroke, heart attack or death was monitored over a
3 -month period and the $\mathrm{ABCD} 2$ scoring system was used to detect the risk of stroke, even though specialist assessment was delayed.

A total of 25 people went on to suffer a stroke, 100 patients suffered a further TIA during the 3-month follow-up and three people died. Commenting on these results, Smith said, "...this rate of stroke was relatively low due to the delay in being able to assess the patients after their initial TIA. Some studies have put the number of people suffering a major stroke within a week of a TIA as high as $10 \%$, which suggests even the seven-day guideline figure may be inadequate."

A number of factors contribute to the delay in assessment of TIA patients. An important factor is the patient not realizing the seriousness of their symptoms. The initial symptoms are temporary, lasting from minutes to hours, and thus patients often feel better by the time they are seen by a clinician.

The researchers conclude that "...if the speed with which TIA patients can be evaluated is improved, many strokes in the UK each year could be prevented".

Source: Selvarajah JR, Smith CJ, Hulme S, Georgiou RF, Vail A, Tyrrell PJ: Prognosis in patients with transient ischemic attack (TIA) and minor stroke attending TIA services in the North West of England: the NORTHSTAR Study. J. Neurol. Neurosurg. Psychiatry doi:10.1136/jnnp.2007.129163 (2007) (Epub ahead of print). 


\section{Abnormally functioning mirror neurons implicated in autism pathology}

The Centers for Disease Control and Prevention estimate that 1.5 million Americans have autism, which is also the fastest growing developmental disability in the USA. Autism typically appears during the first 3 years of life and hinders social interaction and communication skills.

Manzar Ashtari (Children's Hospital of Philadelphia, PA, USA) and colleagues used diffusion tensor imaging to compare the brains of 13 male patients who had been diagnosed with high-functioning autism or Asperger syndrome and who had an IQ greater than 70 with the brains of 12 healthy control adolescents.

The researchers found that the autistic patients had increased gray matter in the regions that control social processing and learning by observation. "Our findings suggest that the inability of autistic children to relate to people and life situations in an ordinary way may be the result of an abnormally functioning mirror neuron system," said Ashtari.

The mirror neuron system was first identified in the macaque monkey, with researchers having identified a similar system in humans. Mirror neurons are active when an individual is performing an action and experiencing an emotion, and when an individual witnesses the same action, emotion and sensation in others. The mirror neuron system also facilitates learning by seeing and doing, empathizing and understanding others intentions.

Ashtari and colleagues also found a relationship between the amount of gray matter in the left parietal lobe and IQ in the control group. This association was not observed in the autistic patients. "In the normal brain, larger amounts of gray matter are associated with higher IQs," Ashtari said. "But in the autistic brain, increased gray matter does not correspond to IQ, because this gray matter is not functioning properly," he explained. Furthermore, decreased gray matter in the right amygdala region was associated with the severity of social impairment in the autistic patients.

"Impairments in these areas are the hallmark of autism spectrum disorders, and this finding may lead to greater understanding of the neurobiological underpinnings of the core features of autism," commented Joel Bregman (Fay J Lindner Centre for Autism, NY, USA), coauthor of the study.

Source: Ashtari M, Bregman J, Nicholls S: Gray matter enlargement in children with high functioning autism and asperger syndrome using a novel method of diffusion based morphometry. Presented at: Annual Meeting of the Radiological Society Of North America. Chicago, IL, USA, 25-30 November 2007.

\section{Peptide therapy inhibiting NF- $\kappa$ B may prevent Parkinson's disease progression}

Parkinson's disease (PD), a disease of old age with a prevalence of one in 100 in people over 60 years of age, is a slow neurodegenerative disease that affects neurons in the substantia nigra. The cause is unknown, with both the environment and genetics being implicated. The degeneration of neurons results in lower levels of the neurotransmitter dopamine, which leads to the characteristic symptoms of PD.

A recent study by Kali Pahan (Rush University Medical Centre, IL, USA) and colleagues reports that peptide therapy may prevent PD progression. At present, treating neurological disorders with proteins, peptide and genes can be risky and painful, since they cannot cross the $\mathrm{BBB}$ and so have to be injected into the brain. The current study reports on a peptide that is administered by intraperitoneal injection. "This could be a new approach to halt disease progression in PD patients," says Pahan.

\section{... peptide therapy may prevent PD progression.'}

The peptide reverses the biochemical, cellular and anatomical changes of $\mathrm{PD}$ in a mouse model by blocking $\mathrm{NF}-\kappa \mathrm{B}$. The level of $\mathrm{NF}-\kappa \mathrm{B}$ is increased in the area of the mid-brain that is affected in PD. Peptides, proteins and certain drugs cannot enter the brain because they cannot cross the BBB. "To overcome this problem, we have added a tag in front of that peptide that is helping the peptide enter into the brain," explains Pahan.

The peptide was shown to inhibit $\mathrm{NF}-\kappa \mathrm{B}$ and associated toxic molecules, protect neurons, normalize levels of neurotransmitters and improve motor function in the mouse model of PD "Now we need to translate this finding to the clinic and test this peptide in patients with PD. If these results can be replicated in PD patients, it would be a remarkable advance in the treatment of this devastating neurodegenerative disease," Pahan said.

Source: Ghosh A, Roy A, Liu X et al:: Selective inhibition of NF- $\kappa B$ activation prevents dopaminergic neuronal loss in a mouse model of Parkinson's disease. Proc. Natl Acad. Sci. USA 104(47), 18754-18759 (2007). 